\title{
Visual stimuli modulate frontal oscillatory rhythms in a cortically blind patient: evidence for top-down visual processing
}

\author{
Eda Tipura $^{\mathrm{a}, \mathrm{b}}$, Alan J. Pegna ${ }^{\mathrm{c}, \mathrm{d}}$, Beatrice de Gelder $^{\mathrm{e}}$, Olivier Renaud ${ }^{\mathrm{a}}$
}

\begin{abstract}
(a) Methodology and Data Analysis, Department of Psychology, University of Geneva, Switzerland
(b) Distance Learning University, Sierre, Switzerland

(c) School of Psychology, The University of Queensland, Brisbane, Australia

(d) Laboratory of Experimental Neuropsychology, Department of Psychology, University of Geneva, Switzerland

(e) Cognitive and Affective Neuroscience Laboratory, University of Maastricht, The Netherlands
\end{abstract}

\section{Corresponding author:}

Eda Tipura

University of Geneva

Methodology and data analysis (MAD) Laboratory

1205 Geneva, Switzerland

Tel.: +41-22 3799179

E-mail: eda.tipura@unige.ch 
Corresponding author : Eda Tipura 


\begin{abstract}
Objective

We investigated neuronal correlates of faces versus non-faces processing in a cortically blind patient (TN) and a group of healthy age-matched controls in order to test electrophysiological correlates of the processing of pertinent stimuli in this patient.

\section{Methods}

An EEG paradigm was used, in which intact and scrambled faces were displayed on a screen. First, time-frequency transforms were conducted on the patients' data alone. These oscillations were then compared to the frontal activity of six control participants.

Results

Post stimulus oscillatory modulations (synchronisation in theta and alpha frequency bands) of both intact and scrambled faces at frontal scalp sites were observed in TN. These modulations were different for correct and incorrect responses. A more important increase in the theta band for incorrect responses was observed. The oscillatory rhythms highlighted in blindsight and in frontal regions differ from the ones observed in control participants.

\title{
Conclusion
}

Despite the destruction of the visual cortex, oscillatory rhythms are not cancelled out but are shifted to anterior regions, revealing the activity of an alternate pathway for residual visual function.

\section{Significance}

The results provide evidence for a top-down cognitive control process in blindsight.

Keywords: EEG, blindsight, event-related spectral perturbation, vision, frontal cortex, frontal midline theta rhythm. 


\section{Introduction}

Faces are among the most relevant stimuli for humans (Itier and Taylor, 2004). The processing of this type of stimuli has been linked to the occipito-temporal network in an fMRI study (Rossion et al., 2003) and to frontal activations in terms of face-selective responses in the macaque (Scalaidhe et al., 1997). A spreading effect of emotional face perception from occipital and temporal to frontal regions has been reported in epileptic patients implanted with depth electrodes (Krolak-Salmon et al., 2004). Localisation studies using fMRI have shown that a region in the extrastriate cortex is more active for faces than other objects (Kanwisher et al., 1997; Rhodes et al., 2004). This region has consequently been labelled the fusiform face area (FFA) in order to emphasise its face specificity (Kanwisher et al., 1997). Studies addressing the dynamics of face processing with electrophysiological techniques have shown that faces elicit a specific early component, the N170, which appears at around $170 \mathrm{~ms}$ and is characterised as a negativity over the posterior temporal region (e.g. Bentin et al.,1996).

More recently, the extent of processing carried out by the nonstriate visual pathway has received much attention. This pathway, involving the superior colliculus, the pulvinar and the amygdala, is hypothesised to be responsible for the processing of visual information when the visual cortex is damaged, such as for example in the case of blindsight (Weiskrantz, 1986). Blindsight, a condition in which the striate cortex (V1) is damaged resulting in cortical blindness, has been described in certain patients. Patients presenting this condition have shown to be able to discriminate visual features, such as motion direction or orientation (Weiskrantz et al., 1995). These observations were first made on more elementary features, such as light spots that were presented in the blind field, subsequently raising the question of whether higher level representations could use this alternate route as well. Interestingly, stimuli conveying emotional information, including faces and bodies, seem to represent a specific category that is processed in blindsight patients (Van den Stock et al., 2014), which has led to the term "affective blindsight". In an initial study, de Gelder et al. (1999) showed that a patient was able to guess above chance level the expression of emotional faces, even though he was unaware of the presence of the stimulus. Another study on a patient $(\mathrm{TN})$ with bilateral V1 destruction (Pegna et al., 2005) showed that emotionally expressive faces elicited brain responses, notably in the right amygdala. This finding is not altogether surprising, as the amygdala is known to be associated with the 
processing of relevant stimuli in the healthy human brain (Sander et al., 2003), highlighting the evolutionary advantage in processing relevant stimuli. In support of this evolutionary trace, responses to faces and emotional faces have been observed as early as $50-90 \mathrm{~ms}$ and $100-130 \mathrm{~ms}$ respectively and lasting until 200ms in surface (e.g. Linkenkaer-Hansen et al., 1998) as well as in intracranial ERP recordings in healthy subjects (e.g. Seeck et al., 1997; Krolak-Salmon et al., 2004). There is also recent interesting evidence of face cells in the pulvinar (Maior et al., 2010) and the superior colliculus (Nguyen et al., 2014), suggesting specific activations for faces beyond the classical striate pathway.

Over the past years, the study of cognitive processes has been widely linked to oscillations modulated by different types of stimuli in the human brain. The frequency content of electro- or magneto-encephalography measures provides key information about oscillations and has been divided in low (delta range, $<3 \mathrm{~Hz}$; theta range, $4-7 \mathrm{~Hz}$ ), mid (alpha range, $8-12 \mathrm{~Hz}$; beta range, $15-25 \mathrm{~Hz}$ ) and high (gamma range, 30 - $120 \mathrm{~Hz}$ ) frequencies (Tallon-Baudry, 2009). In patient TN, mentioned above and who suffered from bilateral cortical blindness, alpha and beta oscillations in frontal regions were observed for fearful and happy face processing between 100 and $400 \mathrm{~ms}$ post-stimulus (Del Zotto et al., 2013). Another hemianopic patient (GY) showed induced-gamma band oscillations linked to stimulus awareness (Schurger et al., 2006). However, overall, there is little evidence on the modulation of oscillatory activity by visual or cognitive tasks in blindsight.

Consequently, the general aim of this study was to explore the electrophysiological correlates of processing of relevant stimuli in the human brain, using time-frequency decompositions.

Particularly, we aimed to identify the manifestation of unconscious processing in a blindsight patient, and compare it to healthy controls. Since it has been shown that blindsight patients may process visual stimuli in the absence of a functional visual cortex, we questioned the electrophysiological correlates of these residual visual capacities. Our aim was to extend the results observed by Del Zotto et al. (2013) in frontal regions to the processing of faces, independently of (facial) emotional expressions.

Three research questions guided our analyses. Firstly, we wanted to determine the presence of a potential oscillatory response to visual stimuli in general, i.e., independently of the stimulus in TN. We expect a modulation of the post-stimulus oscillatory pattern following visual stimuli in this patient. The presence of a specific activation in one of the explored frequency bands would reflect the activity 
of an alternate pathway for visual processing when the visual cortex is not accessible. In this study, post-stimulus activations were explored over frontal regions in three frequency bands (theta, alpha, and beta). As gamma frequencies have been linked to visual awareness, this frequency was not investigated in patient $\mathrm{TN}$, whose vision lacks the subjective qualia associated with conscious seeing. Moreover, this frequency has been shown to appear following involuntary micro eye movements (Yuval-Greenberg et al., 2008) which have not been controlled in TN during the experiment. Finally, the computation of the wavelet coefficients for lower (delta) frequency uses such a large interval of values that the values obtained are probably too much influenced by pre-stimulus values and by the border conditions of the wavelet transform (Misiti et al., 2016). We thus did not treat the delta band.

The second aim was to investigate whether these modulations were distinct as a function of stimulus category (faces/scrambled faces) and accuracy (correct/incorrect responses) in TN. This analysis would reflect behavioural performance in this patient. The pattern of oscillations for the three different frequencies (theta, alpha, beta) associated with (1) faces (vs scrambled faces) and (2) correct (vs incorrect) responses were analysed.

Finally, in order to obtain more information about the time course of brain activations, we then decided to compare the post-stimulus period between TN and the control participants. In an fMRI study, Van den Stock et al. (2014) showed that the amygdala and the superior temporal sulcus were activated during the passive viewing of faces in the same patient (TN). Therefore, this approach would allow us to test whether TN was different from the control participants.

\section{Materials and method}

\subsection{Participants}

TN was a 52 year-old male patient when he suffered from two consecutive strokes in his left and right visual cortices within 36 days of each other. The MRI scans performed at that time are shown in Figure S1 of the Supplementary Material 1. The left hemisphere stroke affected the parietotemporo-occipital cerebral area including the inferior parietal region, the left inferior/medial/superior occipital areas, the calcarine sulcus and the fusiform gyrus whilst the subsequent stroke damaged the inferior and medial occipital areas, the calcarine sulcus and the 
fusiform gyrus. After the first stroke, TN presented with right hemiplegia and transcortical sensory aphasia, which receded rapidly but left him with a persistent right hemianopia. The second stroke caused a loss of the remaining left visual half field.

A neuropsychological assessment performed 2 months after the second stroke showed no cognitive impairment except for slight word-finding difficulties. He was clinically blind and unable to detect movement, colours, or geometrical shapes. He complained of a sensation of complete darkness and was subjectively never aware of any visual stimulation.

At the time of testing, he was 60 years of age and remained unable to detect any visual stimulus. He claimed that he could occasionally detect that "something had happened" when visually stimulated, but this was not confirmed when he was asked to detect the presence or absence of a strong light source or its motion. The presentation of the stimuli on the computer screen was not associated with any systematic awareness of stimulation and TN stated that he could not see when a stimulus appeared. His case history has been reported extensively elsewhere (Pegna et al., 2005; de Gelder et al., 2008; Buetti et al., 2013; Burra et al., 2013). In addition, 8 age-matched men (mean age $=64$ years, $\mathrm{SD}=2.5$ ) participated as controls in this study. Controls were right-handed, and had a normal or a corrected-to-normal vision. Two control participants were excluded from the analysis as they were unable to remain focused on the task and made numerous eye and muscle movements during the EEG recordings, leading to very noisy data.

\subsection{Stimuli and experimental procedure}

The stimuli were isoluminant greyscale images of faces and scrambled faces $\left(25.4 \mathrm{~cd} / \mathrm{m}^{2}\right)$ in which the individual features (eyes, nose and mouth) had been displaced (Fig. 1). Participants were placed in front of the screen at a viewing distance of $100 \mathrm{~cm}$. Each run began with a fixation cross that lasted $600 \mathrm{~ms}$. The test stimulus then appeared for 500ms and was followed by a blank screen that was displayed for 500ms. Participants were asked to press "1" on the keyboard if they saw a face, and "2" if they saw a non-face using the right forefinger and middle finger. Then the next trial appeared. Prior to the experiment proper, a practice session composed of 4 trials was carried out. Once familiarised with the task, the participants started the experiment, which was composed of 200 trials (100 faces and 100 scrambled faces). For TN, the EEG experiment was composed of 100 trials (50 faces and 50 
scrambled faces). TN, who was unaware of the visual stimuli, was informed that faces and non-faces were going to appear on the screen. He was instructed to keep his eyes open and his gaze oriented in front of him and was asked to guess if the stimulus might be a face or a non-face even if he felt his responses were random. A sound indicated the moment he was required to respond $(800 \mathrm{~ms}$ after stimulus onset). Stimulus delivery was self-paced.

The study was accepted by the local ethics committee (University of Geneva) and all participants signed an informed consent before participating. TN's consent form was read to him by his wife prior to his acceptance. The study was performed in agreement with the Declaration of Helsinki.

\subsection{EEG acquisition}

During the experiment, the participants were seated comfortably in a sound-attenuated room, in front of the screen. EEG was recorded using a 128-channel Biosemi Active-Two system for the healthy controls and a 49-channel Biosemi Active-Two system for TN (Amsterdam, Netherlands). We used five additional flat electrodes which were placed on the outer canthi of the eyes, under the right eye and over the ear lobes for healthy controls, in order to capture any eye movements and blinks. TN had four external electrodes which were placed on the outer canthi of the eyes and above and under the right eye. The EEG was continuously recorded with a sampling rate of $1024 \mathrm{~Hz}$ for the healthy controls and $256 \mathrm{~Hz}$ for TN, referenced to electrodes CMS and Cz. Re-referencing was carried out offline to average reference.

\subsection{Signal processing}

Standard processing of EEG data was done offline using the software Brain Vision Analyzer V.2 (Brain Products, Gilching, Germany) and custom Matlab scripts built on the open source EEGLAB toolbox (Delorme and Makeig, 2004; http://sccn.ucsd.edu/eeglab/). Statistical analyses were not performed with the built-in statistical analysis package in EEGLab, since its cluster approach does not allow computing significance tests for the effects included in the design that we tested. The data from the control group were downsampled to $256 \mathrm{~Hz}$. We extracted epochs that were time-locked to the stimulus presentation beginning $400 \mathrm{~ms}$ prior, and $800 \mathrm{~ms}$ after stimulus onset. Epochs with amplitudes exceeding $-100 \mu \mathrm{V}$ to $+100 \mu \mathrm{V}$ were automatically rejected. The remaining trials were 
visually inspected and any remaining electrical or ocular artefacts were removed manually. A spherical spline interpolation was applied to all the bad electrodes.

\subsection{Behavioural analysis}

In order to test whether $\mathrm{TN}$ responded correctly above chance level to stimulus category, the dprime (sensitivity to differences between stimuli) and the bias (response criterion) were calculated on the 200 trials (Macmillan and Creelman, 2005). Hits represent correct responses to faces, correct rejection represent correct responses to scrambled faces, misses represent incorrect responses to faces and false alarms represent incorrect responses to scrambled faces. A permutation test was applied to evaluate the significance of the d-prime (we shuffled 5000 times the responses, while keeping the stimuli fixed).

Previous work on the same patient has shown a higher perceptual sensitivity to bodies, as compared to other stimuli (faces, butterflies, cars, scrambles) (Van den Stock et al., 2014). In this fMRI study, the amygdala and the superior temporal sulcus were activated following the passive exposure to faces and bodies, suggesting a specific processing of faces as well. Here, we tested the difference between correct and incorrect responses for faces and scrambled faces.

\subsection{Time-frequency decompositions}

The spectral energy on pooled electrodes was calculated using a sinusoidal wavelet transform (Delorme and Makeig, 2004), using the squared modulus of the wavelet coefficients: $\left|F_{k}(f, t)\right|^{2}$, where $F_{k}(f, t)$ is the spectral estimate or wavelet coefficient of trial $k$ at frequency $f$ and time $t$. Since we are at the trial level, the energy was extracted for and log-transformed on each trial separately. Averaging these trials would then represent the event-related spectrum (ERS) and if baseline corrected the eventrelated spectral perturbation (ERSP) (Grandchamp and Delorme, 2011).

Three regions of interest of the scalp were defined, representing frontal, right frontal and left frontal scalp sites (see Fig. 2. A and B). Three different frequency bands were analysed: theta $(3.5 \mathrm{~Hz}-8 \mathrm{~Hz})$, alpha $(8 \mathrm{~Hz}-12 \mathrm{~Hz})$ and beta $(12 \mathrm{~Hz}-30 \mathrm{~Hz})$. The energy was first analysed for TN alone, independently of the stimulus category. Then, the post-stimulus baseline corrected spectral energy (see below) was used to include the category of the stimulus and accuracy in the analysis of TN. Finally, a 
comparison between TN and the control participants was carried out. Since control participants responded correctly to almost $100 \%$ of the trials, correct and incorrect responses were used in the last comparisons.

\subsubsection{Post-stimulus spectral changes in $T N$}

To assess whether a post-stimulus activation was present in TN, time-frequency decompositions were applied to the segmented data of all the 81 trials that remained after the signal processing (faces and scrambled faces). We compared the energy of the mean baseline (400ms prestimulus) to the mean post-stimulus period (100-800ms). A paired $t$ test was applied for each frequency band on the pooled electrodes of the three frontal regions.

We also applied current source density estimates for surface potentials (CSD) (Kayser and Tenke, 2006) using the CSD toolbox (http://psychophysiology.cpmc.columbia.edu/Software/CSDtoolbox/) in order to assess whether the activations observed on the electrodes of the three frontal regions are really reflecting the activity of underlying frontal sites. This method calculates the surface Laplacian using spherical splines and minimises the influence of distal sources while increasing the influence of local generators. Among others, it has the advantage to be independent of the choice of reference. Since we applied it to single trials and not event-related potential average, we tested two different spline flexibilities: 4 (rigid spline interpolation) and 8 (very rigid spline interpolation).

\subsubsection{Post-stimulus spectral changes in $\mathrm{TN}$ for faces versus scrambled faces and correct versus incorrect responses}

Since blindsight is a very rare condition and has not been reported extensively in the literature, we followed an exploratory approach in our analysis. Moreover we do not focus on the timing of the differences, but merely on general tendencies in the post-stimulus oscillation pattern. So, to address possible post-stimulus changes linked to the stimulus category and accuracy in $\mathrm{TN}$, we adjusted by least-squares a polynomial regression of degree 2 on the baseline corrected spectral energy between 100 and $800 \mathrm{~ms}$ after the presentation of the stimulus of each trial separately for the three frequency bands. As we tested the differences between categories on the post-stimulus period, the energy was 
baseline corrected (absolute). This approach allows us to condense the post-stimulus activation, based on the increases and decreases of the energy for each condition and each region of interest for an extended period after the presentation of the stimulus. The subjectivity of selecting time intervals (e.g. every $100 \mathrm{~ms}$ ) is therefore avoided, in favour of more informative general tendencies of post-stimulus spectral changes. Moreover, the problem of multiple testing is strongly decreased since there are fewer parameters than the usual number of intervals. Finally, this approach likely possesses more statistical power as it uses the energy at all time points to compute each coefficient. Analysing every time point, as it is often done, would lead to a tremendous number of tests. Therefore, the aim of our approach is to diminish the number of tests we would obtain with a more classical approach, while taking into account the entire post-stimulus period. For a more classical analysis of the EEG data, see the first part of the Supplementary Material 2. The intercept (corresponding to the baseline corrected energy at $450 \mathrm{~ms}$ post-stimulus as we centred the time), the slope (or linear tendency of the post-stimulus energy), and a quadratic effect (signalling a U-shape with a positive coefficient or the inverse of Ushape for a negative coefficient) were calculated. The three parameters were extracted for each trial. They were truncated at 3 standard deviations, to avoid influence of extreme values. We obtained 36 values per trial and per parameter, corresponding to the coefficients associated with each frequency (theta, alpha, beta) for each condition (faces, scrambled faces), each accuracy (correct, incorrect responses) and each scalp region (frontal, left frontal, right frontal). Put another way, there are three parameters (intercept, slope, quadratic) estimated for three frequency bands (theta, alpha, beta), leading to 9 dependent variables (DV). Each DV was submitted to an analysis of variance with three within factors: Condition (Faces/Scrambled faces) x Accuracy (Correct/Incorrect) x Region (Left frontal/Frontal/Right frontal). In order to avoid errors due to multiple testing, we applied the closure principle on each frequency band separately, which controls strongly the FWER (Bretz et al., 2010). In brief, for each frequency band (theta, alpha, beta), each effect (condition, accuracy, region, and their interactions) is tested with a null hypothesis. The effects of the intercept, the slope and the quadratic effect are therefore leading to three null hypotheses, where an effect in the intercept represents the first hypothesis (H1), an effect on the slope represents the second hypothesis (H2) and the quadratic effect represents the third hypothesis $(\mathrm{H} 3)$. Then a multivariate analysis of variance (MANOVA) is applied 
for each pair of hypothesis $(\mathrm{H} 1 \cap \mathrm{H} 2, \mathrm{H} 1 \cap \mathrm{H} 3, \mathrm{H} 2 \cap \mathrm{H} 3)$. Finally, a MANOVA is applied on the 3 DVs together $(\mathrm{H} 1 \cap \mathrm{H} 2 \cap \mathrm{H} 3)$. For a chosen level $\alpha$, a null hypothesis for a given effect can be rejected if all the intersections implying this effect are significant. The same procedure was applied to all the effects and all of the three frequency bands.

As a check, we applied the same procedure to the CSD transformed data with two different spline flexibilities: 4 (rigid spline interpolation) and 8 (very rigid spline interpolation).

\subsubsection{Post-stimulus spectral changes in $T N$ and control participants for faces versus scrambled}

faces

In order to address possible post-stimulus changes linked to the stimulus category in TN versus control participants, the same procedure as above (2.6.2) was applied to the control participant's data: we adjusted a polynomial regression of degree 2 on the baseline corrected spectral energy between 100 and $800 \mathrm{~ms}$ after the presentation of the stimulus of each trial separately for the three frequencies and each subject separately. We obtained 18 values per trial, per subject, and per parameter, corresponding to the coefficients associated with each frequency (theta, alpha, beta) for each condition (faces, scrambled faces) and each scalp region (frontal, left frontal, right frontal). There are three parameters (intercept, slope, quadratic) estimated for three frequency bands (theta, alpha, beta), leading to 9 DVs. For a more classical analysis of the EEG data, see the second part of the Supplementary Material 2. Consequently, to evaluate our hypotheses, 9 multi-level models (Snijders and Bosker, 1999) were performed, with subject as a random effect, condition (faces, scrambled faces) and region (frontal, right frontal, left frontal) as level-one fixed effects and participant (TN, controls) as level-two fixed effect.

As a check, we applied the same procedure to the CSD transformed data with two different spline flexibilities: 4 (rigid spline interpolation) and 8 (very rigid spline interpolation).

\section{Results}

\subsection{Behavioural results}


Control participants responded correctly to the trials with rates of $98.58 \%$ (S1:100\%; S2: 100\%; S3: 98.5\%; S4: 99.5\%; S5: 97.5\%; S6: 96\%), showing that the task was easily carried out and presented no difficulty. TN claimed that he could not see the stimuli but agreed to guess, in his own words, "answering the first thing that came to his mind without thinking". He scored 114 correct responses (guesses) out of 200 presentations with 57 hits, 43 misses, 57 correct rejections and 43 false alarms. He responded correctly above chance level to stimulus category $\left(d^{\prime}=0.35, \mathrm{p}<0.05\right.$ unilateral). The response bias is exactly zero, since he responded to each category at exactly the same rate, showing that he did not favour a response over another.

\subsection{Time-frequency decompositions}

\subsubsection{Post-stimulus spectral changes in $T N$}

Our first aim was to explore whether a post-stimulus response in the time-frequency domain was present in $\mathrm{TN}$, independently of the stimulus category. We observed a significant post-stimulus increase on pooled electrodes in frontal $\left(\mathrm{t}(80)=6.329, \mathrm{p}<10^{-5}\right)$, right frontal $\left(\mathrm{t}(80)=4.236, \mathrm{p}<10^{-4}\right)$ and left frontal $\left(\mathrm{t}(80)=3.842, \mathrm{p}<10^{-4}\right)$ scalp sites in the theta frequency band. These increases can be viewed on the energy on Fig. 3. A, B and C. A significant increase was observed in frontal $(\mathrm{t}(80)=2.949, \mathrm{p}=0.004)$ and left frontal $(\mathrm{t}(80)=2.152, \mathrm{p}=0.034)$ scalp sites in the alpha band as well (Fig. 3. A, C). For the beta frequency band no significant post-stimulus activation was observed (Fig. 3. A, B, C). Even when using a very conservative Bonferroni correction over these nine tests, the three tests associated with the theta band remain highly significant (see table 1).

The same pattern of significance is observed (not shown) after the CSD transform when the spline flexibility was set to 8 (very rigid), strengthening the interpretation of the involvement of frontal regions on the observed modulations. With a lower spline flexibility (set to 4), results are less significant (not shown), probably due to the large wiggliness of single trials (CSD are most often applied on average ERPs).

\subsubsection{Post-stimulus spectral changes in $\mathrm{TN}$ for faces versus scrambled faces and correct versus}

\section{incorrect responses}

Since post-stimulus spectral changes were observed for all kinds of stimuli in TN, our second aim was to specify the characteristics of these changes. We therefore tested the differences for faces as 
compared to scrambled faces and for correct as compared to incorrect responses in TN.

Results of the nine ANOVAs are summarised in table 2, where only significant p-values are displayed. For the factor Accuracy, a significant difference is present in the intercept of the theta band and in the slope of the theta and alpha bands. The interaction between the condition and the accuracy is significant in the quadratic effect of the theta band. The Figure 4 represents the evolution of the energy over time for the theta (A), alpha (B) and beta (C) bands. As we can see on this figure, incorrect responses lead to higher coefficients than correct responses in the theta band, especially for faces. In the alpha band, incorrect responses also lead to higher coefficients than correct responses. The quadratic term does not seem to better represent TN's EEG data, as represented in Fig. 4. Since this procedure is also applied to the control's data in the section 2.6.3, the quadratic effect was kept on the patients' data. Adjusting only the intercept and the linear effect (without the quadratic effect) leads to the same results for these two parameters. The number of trials per category was the following: 23 Correct faces, 23 Correct scrambled faces, 19 Incorrect faces, 16 Incorrect scrambled faces.

\subsubsection{Post-stimulus spectral changes in $\mathrm{TN}$ and control participants for faces versus scrambled}

\section{faces}

Our third aim was to test the differences between the conditions (faces versus scrambled faces) and between the subjects (TN versus control participants). Results of the nine multi-level models are summarised in table 3 , where only significant p-values are displayed. Since we are adopting an exploratory approach, we will not interpret all of the p-values. Instead, we will highlight the interesting effects where the conditions differ and where $\mathrm{TN}$ is significantly different from control participants. These differences can be observed in principal effects as well as in the interactions between the participants (TN versus controls) or the conditions (faces versus scrambled faces) with other factors.

In the theta band, significant effects for the intercept, the slope and the quadratic effect are observed. Concerning the intercept, after correction for multiple comparisons, an effect of the participant (TN versus controls) was observed, with TN showing higher post-stimulus activations than control participants (see Fig. 5.A). For the intercept, the interaction region* participant is also significant, again suggesting a difference between TN and controls. The slope of the theta band is 
significantly higher for $\mathrm{TN}$, as compared to control participants before, but not after correction for multiple comparisons (see Fig. 5.B). Finally, a difference between TN and control participants is found in the interaction region* participant for the quadratic effect (see Fig. 5.C). Indeed, control participants are characterised by a more positive curve than $\mathrm{TN}$ in the theta band.

In the alpha band, TN's intercept is significantly higher from control participants before but not after correction for multiple comparisons. The interaction region* participant is significant for the same parameter (see Fig. 5D). As for the slope, the interaction condition * participant is significant before but not after correction, suggesting a difference between $\mathrm{TN}$ and control participants when including the condition (see Fig. 5.E).

In the beta band, the interaction region * participant is significant before but not after correction for multiple comparisons.

Finally, after the CSD transform, the patterns of activations are very similar both for the spline flexibility set to 4 and 8 . The post-stimulus energy is following the same direction for each frequency band (increase or decrease) over time, the interaction plots (Fig. 5) are very similar, and the highly significant p-values are still significant after the transformation, suggesting that the observed effects are reflecting the involvement of frontal sources.

\section{Discussion}

The aim of our study was to examine the oscillatory brain responses of a cortically blind person (TN) using human faces opposed to (scrambled) non-faces, and to compare his patterns of activation to those of a group of control participants, building on previous observations showing modulations in alpha and beta frequency bands over frontal regions by facial emotional expressions in this patient (Del Zotto et al., 2013). Three main findings were evidenced: oscillatory rhythms were modulated by visual stimuli in TN, differences in oscillatory responses were observed between TN and control participants, and differences in oscillatory responses were found across stimulus categories and accuracy in TN. The results of TN have to be interpreted with caution, since a training effect due to the repeated investigations in this patient may occur and the amount of used epochs per condition in the analysis including correct and incorrect responses in TN is low. All comparisons with control 
participants should also be interpreted with caution, since their data were recorded with different electrode montages.

Our initial aim was to explore whether an activation was present in patient TN in frontal regions and in low frequency bands. This analysis was performed independently of the stimulus category, in order to test whether a visual response of any kind would appear. A significant poststimulus increase in the theta band was observed in the three regions of interest, as well as an increase in the alpha frequency band in frontal and left frontal regions, but the latter did not survive the correction for multiple comparisons. These results provide electrophysiological support for blindsight, showing that despite V1 damage and cortical blindness, modulation of brain activity arises through visual stimulation. Responses in the theta band have been observed in tasks involving working memory (e.g. Jensen and Tesche, 2002) and in attentional cognitive tasks (Basar and Güntekin, 2012). The frontal midline theta rhythm has been widely studied in MEG and EEG paradigms and has been related to mental tasks (Inanaga, 1998), such as for example mental calculation (Mizuhara et al., 2004). Combining EEG and fMRI, these latter authors suggested that theta oscillations reflect the activity of a long-range cortical network during a mental task, involving the anterior midline, the posterior cingulate and the right middle temporal cortices. This rhythm also seems to reflect encoding and retrieval of new information (Klimesch et al., 1996; Klimesch et al., 1997) and sustains the activity of the P300 wave in the perception of novel stimuli (Dietl et al., 1999). A rapid and brief increase in the power of the theta band (125-250ms) has been shown in a word recognition memory task, raising a possible link between hippocampal involvement in episodic memory and theta synchronisation (Burgess and Gruzelier, 1997). Moreover, this frequency band seems to appear in response to emotional expression with high arousal in healthy subjects (Balconi and Lucchiari, 2006). In this latter study, participants were shown pictures of faces expressing either positive, negative, or neutral facial expressions. An event-related desynchronisation in the theta band in anterior regions was interpreted by the authors as reflecting the emotional significance of faces. In another investigation, modulations of low alpha and faster beta oscillations in right and left frontal sites were observed for emotional expressions in the same patient examined in our current study (TN) (Del Zotto et al., 2013). 
However, theta modulations have never been shown in the case of cortical blindness. Our study is therefore the first evidence to demonstrate responses in the low frequencies for visual stimulation and in frontal regions in cortical blindness. We hypothesise that the post-stimulus increase observed in TN in the theta band may reflect mental involvement and sustained attention during the experiment.

In the second part of our analyses, we included the category of the stimulus (faces versus scrambled faces) and accuracy (correct versus incorrect responses) in TN. Interesting effects were highlighted in the theta and the alpha frequency bands. In the theta band, an effect of accuracy is present where incorrect responses led to higher coefficients than correct responses. The interaction condition $\mathrm{x}$ accuracy was also significant, with faces leading to greater coefficients for incorrect responses than correct responses, and scrambled faces leading to higher coefficients for correct responses than for incorrect responses. Frontal theta power has been shown to be elicited by uncertainty following a response in an Eriksen flanker task where participants had to discriminate a target letter in a string composed of 7 letters, and in a circle discrimination task where participants had to discriminate the size of a circle (Selimbeyoglu et al., 2012). The trials varied as a function of discrimination complexity, and the responses leading to high uncertainty were followed by a less important total theta power than the other conditions. The frontal midline theta rhythm has also been linked to cognitive control. In a study, Cavanagh et al. (2009) used a Flanker task to test this hypothesis. The increase in theta activity is suggested to reflect the relations between the medial prefrontal cortex associated with action-monitoring following error trials and the lateral prefrontal cortex. The authors interpret this theta synchronisation as a process of cognitive control following an error. We therefore hypothesise that the increase in the theta band and in frontal sites following stimuli leading to incorrect responses in TN may reflect a top-down cognitive control process. These results may seem contradictory with the results Del Zotto et al. (2013) found in the same patient, where increases in alpha and beta bands were observed following the presentation of emotional faces. This discrepancy is certainly due to the type of stimuli used. While Del Zotto et al. (2013) used emotional faces, we used neutral faces with the aim to extract responses to pertinent stimuli in general (independently of emotion). Therefore, we may postulate that lower frequencies such as theta are 
representative of pertinence whereas higher frequencies (in this case alpha and beta) would reflect emotion processing.

Finally, a comparison in the oscillatory changes in $\mathrm{TN}$ as compared to control participants was made. Results show a difference between $\mathrm{TN}$ and control participants in theta and alpha frequency bands. These differences can be observed in the principal effects and in their interactions with other factors. In the theta band, higher intercepts are observed in $\mathrm{TN}$, as compared to controls, meaning that at $450 \mathrm{~ms}$ post-stimulus, the increase in the theta band is higher for TN. As for the slope of the same frequency band, TN is represented by higher slope coefficient than control participants, meaning that during the post-stimulus period, the energy of this frequency is showing a more prominent increase over time for TN. As discussed above, there is experimental evidence that theta oscillations are related to cognitive processing. A dissociation between theta and alpha band activations and memory demands has been highlighted by Klimesch (1996), where episodic memory was sustained by theta oscillations while alpha oscillations reflected long-term memory. Moreover, this frequency band has been linked to selective attention in an oddball paradigm task (Basar-Eroglu et al., 1992) 300ms after the presentation of the deviant stimulus. This indicates a sustained activity over later post-stimulus periods, which is consistent with the increase of this activity in TN (reflected by the theta slope), therefore suggesting the involvement of an attentional load in the processing of both faces and scrambled faces.

The slope of the alpha band provides an interesting result, where TN shows higher increase of activations than control participants, especially for faces. The alpha frequency band was first linked to "cortical idling" (Pfurtscheller et al., 1996), but there is now some evidence that certain types of tasks (or stimuli) can modulate these oscillations. Changes in the alpha frequency-band have been observed in paradigms involving an attentional component, especially in covert attention shifting (Posner et al., 1980). In terms of frequency-band activity, some studies have found a decrease in the alpha activity during the attention shift occurring in posterior areas, persisting until the target appeared (Yamagishi et al., 2005) and occurring at sites contralateral to the attended visual stimulus. These results suggest that a shift of attention is associated with excitability occurring in the contralateral posterior cortex, thus facilitating the processing of attended stimuli. Remarkably, other studies (e.g. Worden et al., 
2000) have found an inverse effect, where an increase in the alpha band ipsilateral to the attended position was observed. This effect has been interpreted as an inhibitory process for unattended positions. These observations were made in posterior regions, as opposed to our study, in which the increase in this frequency-band was observed over frontal sites. Recent evidence shows that frontal alpha synchronisation is linked to internal and creative thinking (Fink et al., 2009; Fink and Benedek, 2014), which is defined as the generation of original and creative contributions in problem solving and in cognitive processing in general. In their study, Fink et al. (2009) elaborated a task where participants had to imagine unusual uses of different objects. Higher original responses were associated with more important alpha synchronisation in frontal sites. Since TN was not able to consciously perceive the stimuli, he was instructed to guess the presence of a face or a non-face. We may suppose the involvement of some type of creative thinking in the processing of faces when they are not consciously perceived. In patient $\mathrm{TN}$, a frontal activity was found in the case of mental imagery, i.e. when he was asked to imagine a face (de Gelder et al., 2014). Indeed, frontal, temporal and parietal regions were activated when $\mathrm{TN}$ was instructed to imagine a person (versus a tree), suggesting that the striate cortex is not necessary for mental imagery. The resolution of ambiguity is an important facet of creativity and has been studied using the ambiguity resolution task and ambiguous words (Atchley et al., 1999). During the whole experiment, TN was put in an ambiguous context and the alpha activity in frontal regions might reflect the resolution of this ambiguity when faces were displayed on the screen. Taken together, these observations point out the implication of a top-down processing in blindsight, which seems to be modulating the alpha oscillations in frontal scalp sites. In the absence of an intact striate cortex, processing of visual information is therefore shifted to anterior regions through low wave oscillations, leading to a top-down effect for pertinent stimuli. This corroborates the electrophysiological results and shows that different regions respond to these stimuli in blindsight, and suggests the implication of a distinct network in this response than the one controls would use.

The study of brain-damaged patients gives the opportunity to explore the alternative routes involved in the processing of relevant and non-relevant stimuli. When studying cognitive and affective phenomena in the brain-damaged patients, the question that arises is the generalisation of the 
conclusions in healthy subjects. Indeed, transposing results from a patient to a healthy participant requires caution, since in blindsight patients, experience-dependent plasticity involving the abovementioned structures may occur (Tamietto and de Gelder, 2010). Transcranial magnetic resonance was used in one study (Jolij and Lamme, 2005) to imitate the effects of a blind field in healthy subjects. When applied over the occipital cortex, TMS induces cortical blindness, allowing the study of the processing of emotional stimuli in healthy participants when the V1 route was not accessible. In this study, participants were able to guess sad and happy expressions, without explicit knowledge of the stimuli, suggesting that non-conscious perception of emotional stimuli may involve an alternative route in healthy controls as well.

\section{Conclusions}

In summary, our results support the existence of a different pathway for processing visual stimuli in blindsight. Given the poor representation of studies integrating blindsight patients in the literature and the difficulty to deal with such data, we followed an exploratory approach in our analyses. The idea was to condense the post-stimulus oscillatory changes while avoiding errors due to multiple comparisons. Altogether, our findings suggest specific oscillatory changes linked to visual stimuli in a blindsight patient, as shown by the low frequencies appearing after the presentation of the stimuli. These results can be interpreted most likely as reflecting the involvement of a non-striate network in processing visual stimuli in TN. This appears to integrate a complex cognitive process involving memory and attention, as well as a top-down elaboration, reflected in the midline frontal theta rhythm. 


\section{Conflict of interest}

None of the authors have potential conflicts of interest to be disclosed.

\section{Acknowledgments}

The authors thank Cyril Pernet for help and advice about the Laplacian transforms, Arnaud Delorme for help and support with the EEGLab toolbox and Andres Posada for technical support.

The research was supported by the Swiss National Science Foundation (grant no. 320030-144187), which had no role in study design, the collection, analysis and interpretation of data, the writing of the report and the decision to submit the article for publication. 


\section{References}

Atchley, R.A., Keeney, M., \& Burgess, C. (1999). Cerebral hemispheric mechanisms linking ambiguous word meaning retrieval and creativity. Brain Cogn, 40(3), 479-499. http://dx.doi.org/10.1006/brcg.1999.1080.

Balconi, M \& Lucchiari, C. (2006). EEG correlates (event-related desynchronization) of emotional face elaboration: a temporal analysis. Neurosci Lett, 392 (1), 118-123. http://dx.doi.org/10.1016/j.neulet.2005.09.004.

Basar, E., \& Güntekin, B. (2012). A short review of alpha activity in cognitive processes and in cognitive impairment. Int J Psychophysiol, 86(1), 25-38. http://dx.doi.org/10.1016/j.ijpsycho.2012.07.001.

Başar-Eroglu, C., Başar, E., Demiralp, T., \& Schürmann, M. (1992). P300-response: possible psychophysiological correlates in delta and theta frequency channels. A review. Int $J$ Psychophysiol, 13(2), 161-179. http://dx.doi.org/10.1016/0167-8760(92)90055-G.

Bentin, S., Allison, T., Puce, A., Perez, E., \& McCarthy, G. (1996). Electrophysiological Studies of Face Perception in Humans. J Cogn Neurosci, 8(6), 551-565. http://dx.doi.org/10.1162/jocn.1996.8.6.551.

Bretz, F., Hothorn, T., \& Westfall, P. (2010). Multiple Comparisons Using R. Boca Raton, FL: Chapman and Hall/CRC.

Buetti, S., Tamietto, M., Hervais-Adelman, A., Kerzel, D., de Gelder, B., \& Pegna, A.J. (2013). Dissociation between Goal-directed and Discrete Response Localization in a Patient with Bilateral Cortical Blindness. J Cogn Neurosci, 25(10), 1769-1775. http://dx.doi.org/10.1162/jocn_a_00404.

Burgess, A.P., \& Gruzelier, J.H. (1997). Short duration synchronisation of human theta rhythm during recognition memory. Neuroreport, 8(4):1039-1042.

Burra, N., Hervais-Adelman, A., Kerzel, D., Tamietto, M., de Gelder, B., Pegna, A.J. (2013). Amygdala activation for eye contact despite complete cortical blindness. $J$ Neurosci,33(25), 10483-10489. http://dx.doi.org/ 10.1523/JNEUROSCI.3994-12.2013. 
Cavanagh, J.F., Cohen, M., \& Allen, J.J.B. (2009). Prelude to and Resolution of an Error: EEG Phase Synchrony Reveals Cognitive Control Dynamics during Action Monitoring. J Neurosci, 29(1), 98105. http://dx.doi.org/10.1523/JNEUROSCI.4137-08.2009.

de Gelder, B., Vroomen, J., Pourtois, G., \& Weiskrantz, L. (1999). Non-conscious recognition of affect in the absence of striate cortex. Neuroreport, 10(18), 3759-3763. http://dx.doi.org/ 10.1097/00001756-199912160-00007.

de Gelder, B., Tamietto, M., van Boxtel, G., Goebel, R., Sahraie, A., Van den Stock, J., Stienen, B. M. C., Weiskrantz, L., \& Pegna, A. (2008). Intact navigation skills after bilateral loss of striate cortex. Curr Biol, 18(24), R1128-R1129. http://dx.doi.org/10.1016/j.cub.2008.11.002.

de Gelder, B., Tamietto, M., Pegna, A., \& Van den Stock, J. (2014). Visual imagery influences brain responses to visual stimulation in bilateral cortical blindness. Cortex, 72, 15-26. http://dx.doi.org/10.1016/j.cortex.2014.11.009.

Delorme, A., \& Makeig, S. (2004). EEGLAB: an open source toolbox for analysis of single-trial EEG dynamics including independent component analysis. J Neurosci Methods, 134(1), 9-21. http://dx.doi.org/10.1016/j.jneumeth.2003.10.009.

Del Zotto, M., Deiber, M.-P., Legrand, L., de Gelder, B., Pegna, A.J. (2013). Emotional expressions modulate low $\alpha$ and $\beta$ oscillations in a cortically blind patient. Int J Psychophysiol, 90(3), 358-362. http://dx.doi.org/10.1016/j.ijpsycho.2013.10.007.

Dietl, T., Dirlich, G., Vogl, L., Lechner, C., \& Strian, F. (1999). Orienting response and frontal midline theta activity: a somatosensory spectral perturbation study. Clin Neurophysiol, 110, 12041209.

Fink, A., Grabner, R.H., Benedek, M., Reishofer, G., Hauswirth, V., Fally, M., Neuper, C., Ebner, F., \& Neubauer, A.C. (2009). The creative brain: Investigation of brain activity during creative problem solving by means of EEG and FMRI. Hum Brain Mapp, 30(3), 734-748. http://dx.doi.org/10.1002/hbm.20538.

Fink, A., \& Benedek, M. (2014). EEG alpha power and creative ideation. Neurosci Biobehav Rev, 44(100), 111-123. http://dx.doi.org/10.1016/j.neubiorev.2012.12.002. 
Grandchamp, R., \& Delorme, A. (2011). Single-trial normalization for event-related spectral decomposition reduces sensitivity to noisy trials. FrontPsychol, 2(236), 1-14.

Inanaga, K. (1988). Frontal midline theta rhythm and mental activity. Psychiatry Clin Neurosci, 52, $555-566$.

Itier, R. J., \& Taylor, M. J. (2004). N170 or N1? Spatiotemporal differences between object and face processing using ERPs. Cereb Cortex, 14(2), 132-142. http://dx.doi.org/10.1093/cercor/bhg111.

Jensen, O., \& Tesche, C.D. (2002). Frontal theta activity in humans increases with memory load in a working memory task. Eur J Neurosci, 15(8), 1395-1399. http://dx.doi.org/10.1046/j.14609568.2002.01975.x.

Jolij, J., \& Lamme, V. A. F. (2005). Repression of unconscious information by conscious processing: Evidence from affective blindsight induced by transcranial magnetic stimulation. Proc Natl Acad Sci U S A, 102(30), 10747-10751. http://dx.doi.org/10.1073/pnas.0500834102.

Kanwisher, N., McDermott, J., \& Chun, M. M. (1997). The Fusiform Face Area: A Module in Human Extrastriate Cortex Specialized for Face Perception, J Neurosci, 17(11), 4302- 4311.

Kayser, J., \& Tenke, C.E. (2006). Principal components analysis of Laplacian waveforms as a generic method for identifying ERP generator patterns: I. Evaluation with auditory oddball tasks. Clin Neurophysiol, 117(2), 348-368. http://dx.doi.org/10.1016/j.clinph.2005.08.034.

Klimesch, W. (1996). Memory processes, brain oscillations and EEG synchronisation. Int J Psychophysiol, 24(1-2), 61-100. http://dx.doi.org/10.1016/S0167-8760(96)00057-8.

Klimesch, W., Doppelmayer, M., Russegger, H., \&Pachinger, T. (1996). Theta band power in the human scalp EEG and the encoding of new information. Neuroreport, 7, 1235-1240.

Klimesch, W., Doppelmayer, M., Schimke, H., \& Ripper, B. (1997). Theta synchronisation and alpha desynchronisation in a memory task. Psychophysiology, 34, 169-176.

Krolak-Salmon, P., Hénaff, M.-A., Vighetto, A., Bertrand, O., \& Mauguière, F. (2004). Early amygdala reaction to fear spreading in occipital, temporal, and frontal cortex: a depth electrode ERP study in human. Neuron, 42(4), 665-676. http://dx.doi.org/10.1016/S0896-6273(04)00264-8. Linkenkaer-Hansena, K., Palvac, J.M., Samsd, M., Hietanend, J.K., Aronene, H.J., \& Ilmoniemia, R.J. (1998). Face-selective processing in human extrastriate cortex around $120 \mathrm{~ms}$ after stimulus onset 
revealed by magneto- and electroencephalography. Neurosci Lett, 253(3), 147-150. http://dx.doi.org/10.1016/S0304-3940(98)00586-2.

Major, Hori, E., Tomaz, C., Ono, T., \& Nishijo, H. (2010). The monkey pulvinar neurons differentially respond to emotional expressions of human faces. Behav Brain Res, 215(1), 129-135. http://dx.doi.org/10.1016/j.bbr.2010.07.009.

Macmillan, N.A., \& Creelman, C.D. (2005). Detection theory: a user's guide - 2nd edition. New Jersey: Lawrence Erlbaum Associates.

Misiti, M., Misiti, Y., Oppenheim, G., \& Poggi J.-M. (2016). Wavelet toolbox user's guide. Natick: The MathWorks, Inc.

Mizuhara, H., Wang, L.Q., Kobayashi, K., \& Yamaguchi, Y. (2004). A long-range cortical network emerging with theta oscillation in a mental task. Neuroreport, 15(8), 1233-1238.

Nguyen, M.N., Matsumoto, J., Hori, E., Maior, R.S., Tomaz, C., Tran, A.H., Ono, T., \& Nishijo, H. (2014). Neuronal responses to face-like and facial stimuli in the monkey superior colliculus. Front Behav Neurosci, 8(85), 1-18. http://dx.doi.org/10.3389/fnbeh.2014.00085.

Pegna, A. J., Khateb, A., Lazeyras, F., \& Seghier, M. L. (2005). Discriminating emotional faces without primary visual cortices involves the right amygdala. Nat Neurosci, 8(1), 24-25. http://dx.doi.org/10.1038/nn1364.

Pfurtscheller, G., Stancak, A, Jr., Neuper, C. (1996). Event-related synchronisation (ERS) in the alpha band - an electrophysiological correlate of cortical idling: A review. Int J Psychophysiol, 24(12), 39-46. http://dx.doi.org/10.1016/S0167-8760(96)00066-9.

Posner, M. I., Snyder, C. R. R., \& Davidson, B.J. (1980). Attention and the Detection of Signals. J Exp Psychol, 109(2), 160-174. http://dx.doi.org/10.1037/0096-3445.109.2.160.

Rhodes, G., Byatt, G., Michie, P. T., \& Puce, A. (2004). Is the Fusiform Face Area Specialized for Faces, Individuation, or Expert Individuation? J Cogn Neurosci, 16(2), 189- 203. http://dx.doi.org/10.1162/089892904322984508.

Rossion, B., Caldara, R., Seghier, M., Schuller, A.-M., Lazeyras, F., \& Mayer, E. (2003). A network of occipito-temporal face-sensitive areas besides the right middle fusiform gyrus is necessary for normal face processing. Brain, 126(11), 2381-2395. 
Sander, D., Grafman, J., \& Zalla, T. (2003). The human amygdala: an evolved system for relevance detection. Rev Neurosci, 14(4), 303-316.

Scalaidhe, S.P.O., Wilson, F.A.W., \& Goldman-Rakic, P.S. (1997). Areal Segregation of FaceProcessing Neurons in Prefrontal Cortex. Science, 278(5340), 1135-1138.

Schurger, A., Cowey, A., \& Tallon-Baudry, C. (2006). Induced gamma-band oscillations correlate with awareness in hemianopic patient GY. Neuropsychologia, 44(10), 1796-1803. http://dx.doi.org/10.1016/j.neuropsychologia.2006.03.015.

Seeck, M., Michel, C.M., Mainwaring, N., Cosgrove, R., Blume, H., Ives, J., Landis, T., \& Schomer, D.L. (1997). Evidence for rapid face recognition from human scalp and intracranial electrodes. Neuroreport, 8(12), 2749-2754. http://dx.doi.org/10.1097/00001756-199708180-00021.

Selimbeyoglua, A., Keskin-Ergen, Y., \& Demiralp, T. (2012). What if you are not sure? Electroencephalographic correlates of subjective confidence level about a decision. Clin Neurophysiol, 123(6), 1158-1167.

Snijders, T.A.B., \& Bosker, R.J. (1999). Multilevel analysis. London: Sage Publications.

Tallon-Baudry, C. (2009). The roles of gamma-band oscillatory synchrony in human visual cognition. Front Biosci (Landmark Ed), 14, 321-332.

Tamietto, M., \& Gelder, B. de. (2010). Neural bases of the non-conscious perception of emotional signals. Nat Rev Neurosci, 11(10), 697-709. http://dx.doi.org/10.1038/nrn2889.

Van den Stock, J., Tamietto, M., Zhan, M., Heinecke, A., Hervais-Adelman, A., Legrand, L. B., ... de Gelder, B. (2014). Neural correlates of body and face perception following bilateral destruction of the primary visual cortices. Front Behav Neurosci, 8(30), 1-10. http://dx.doi.org/10.3389/fnbeh.2014.00030.

Weiskrantz, L. (1986). Blindsight: A case study and implications. Oxford: Oxford University Press.

Weiskrantz, L., Barbur, J. L., \& Sahraie, A. (1995). Parameters affecting conscious versus unconscious visual discrimination with damage to the visual cortex (V1). Proc Natl Acad Sci U S A, 92(13), 6122-6126. 
Worden, M. S., Foxe, J. J., Wang, N., \& Simpson, G. V. (2000). Anticipatory biasing of visuospatial attention indexed by retinotopically specific alpha-band electroencephalography increases over occipital cortex. J Neurosci, 20(6), RC63.

Yamagishi, N., Goda, N., Callan, D. E., Anderson, S. J., \& Kawato, M. (2005). Attentional shifts towards an expected visual target alter the level of alpha-band oscillatory activity in the human calcarine cortex. Brain Res Cogn Brain Res, 25(3), 799- 809.

http://dx.doi.org/10.1016/j.cogbrainres.2005.09.006.

Yuval-Greenberg, S., Tomer, O., Keren, A.S., Nelken, I., \& Deouell, L.Y. (2008). Transient Induced Gamma-Band Response in EEG as a Manifestation of Miniature Saccades. Neuron, 58(3), 429441. 


\section{Figure Legends}

Fig. 1. Stimuli. Stimuli used in the study; A. Faces, B. Scrambled faces.

Fig. 2. Electrodes used in the analyses. Scalp distributions of the 49 electrodes for TN (A) and the 128 electrodes for control participants (B). The black lines represent the three regions of interest (left frontal, frontal, right frontal) and the six electrodes used for the analyses within these regions are highlighted with black dots.

Fig. 3. Event-related spectral perturbation plots. ERSP plots of pooled electrodes in A. frontal, B. right frontal and C. left frontal scalp sites in TN. Stimulus onset is at $0 \mathrm{~ms}$. The left panels represent the mean baseline power spectrum.

\section{Fig. 4. Coefficients associated to the adjusted polynomial regression in TN for the three}

frequency bands. Evolution of the spectral energy according to the three adjusted parameters (intercept, slope, quadratic effect) in TN for (A) the theta band, (B) the alpha band, (C) the beta band as a function of stimulus category (faces/scrambled faces) and accuracy (correct/incorrect responses). Vertical bars represent confidence intervals of $95 \%$ around the central value.

Fig. 5. Evolution of the spectral energy according to the three parameters. Interaction plots representing the evolution of the spectral energy for the different effects between 100 and $800 \mathrm{~ms}$ poststimulus: the intercept in the theta band (A), the slope of the theta band (B), the quadratic effect in the theta band (C), the intercept of the alpha band (D), and the slope of the alpha band (E). The effects are plotted as a function of scalp site $(\mathrm{LF}=$ left frontal, $\mathrm{F}=$ frontal, $\mathrm{RF}=$ right frontal), condition (Faces, Scrambled faces) and participant (TN, Controls). 
Figure 1

A
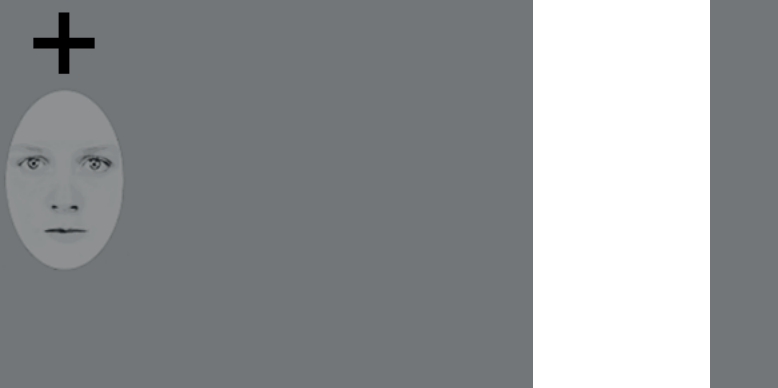
A

Frontal

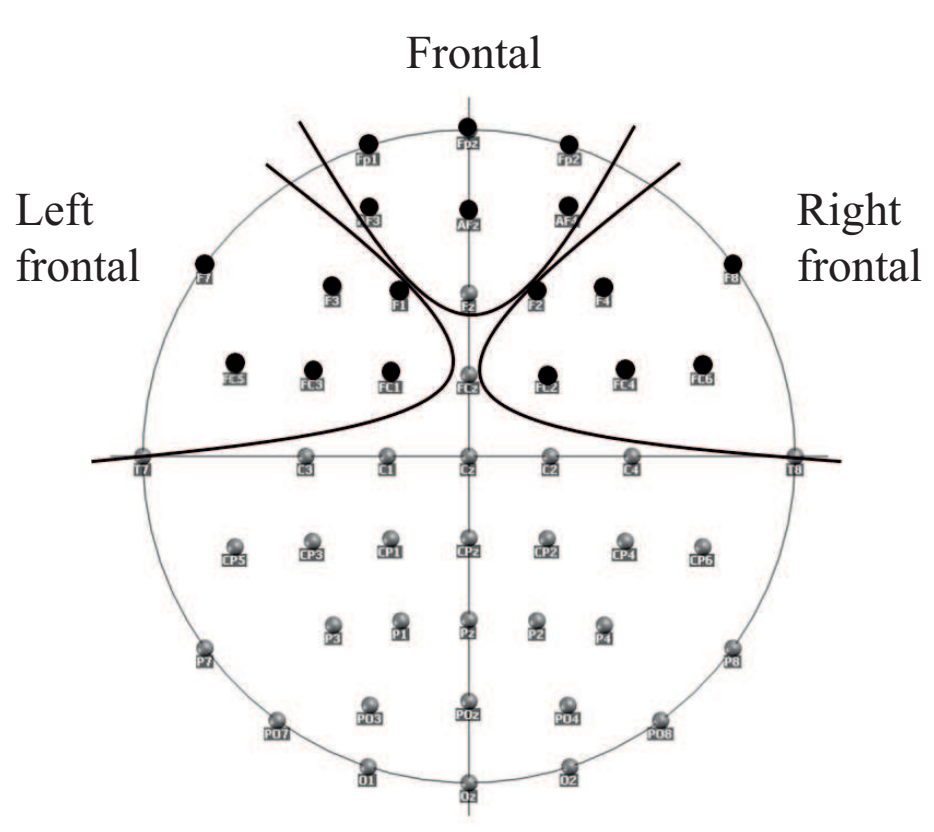

B

Frontal

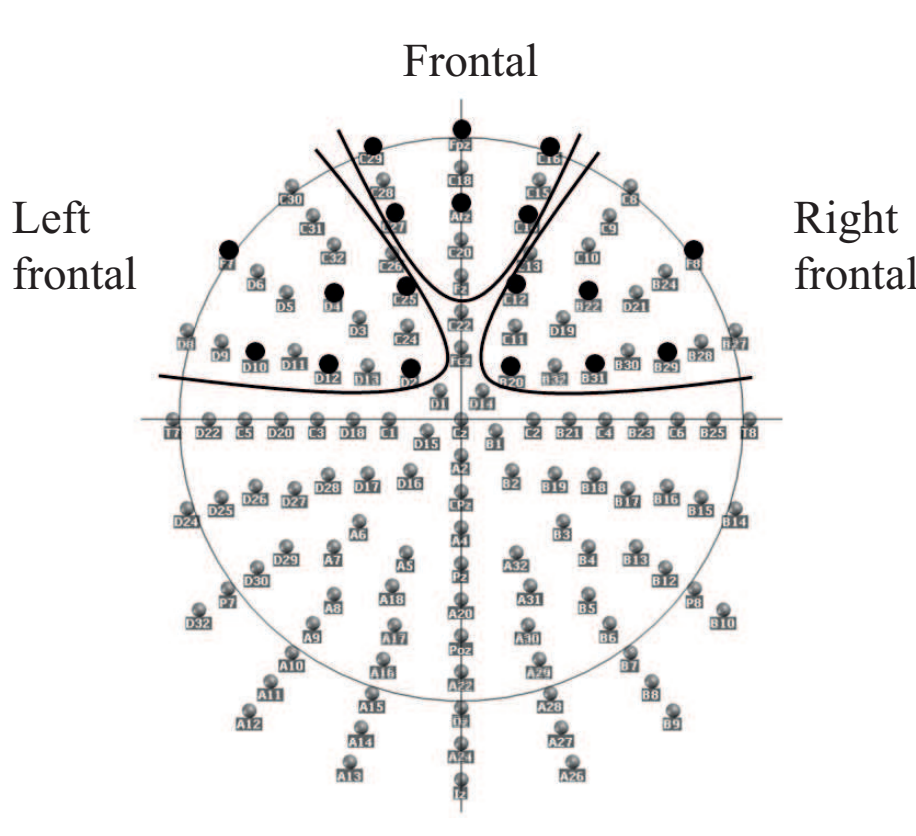

B

Figure 2 
Figure 4

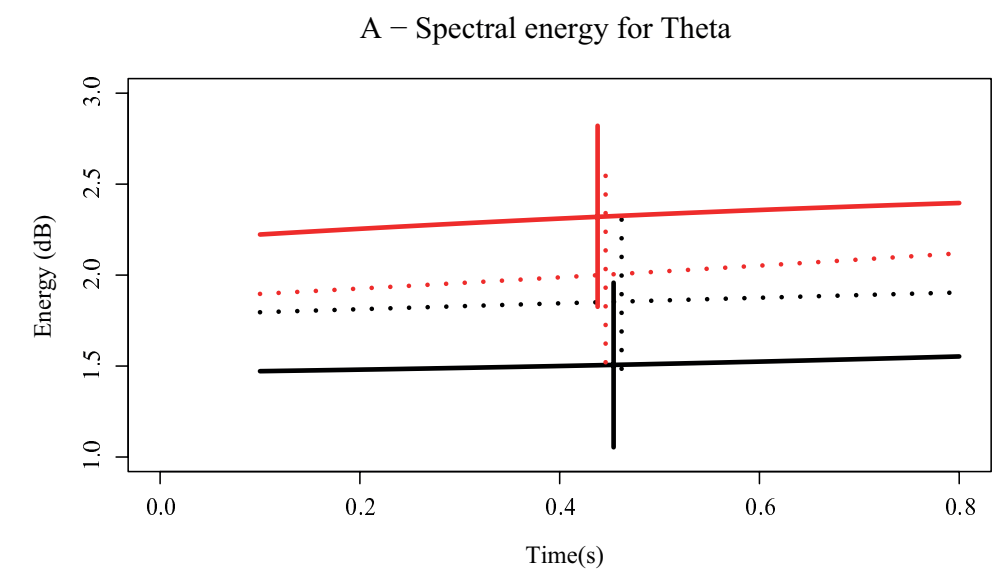

Faces incorrect

Scrambled faces incorrect

Faces correct

......... Scrambled faces correct
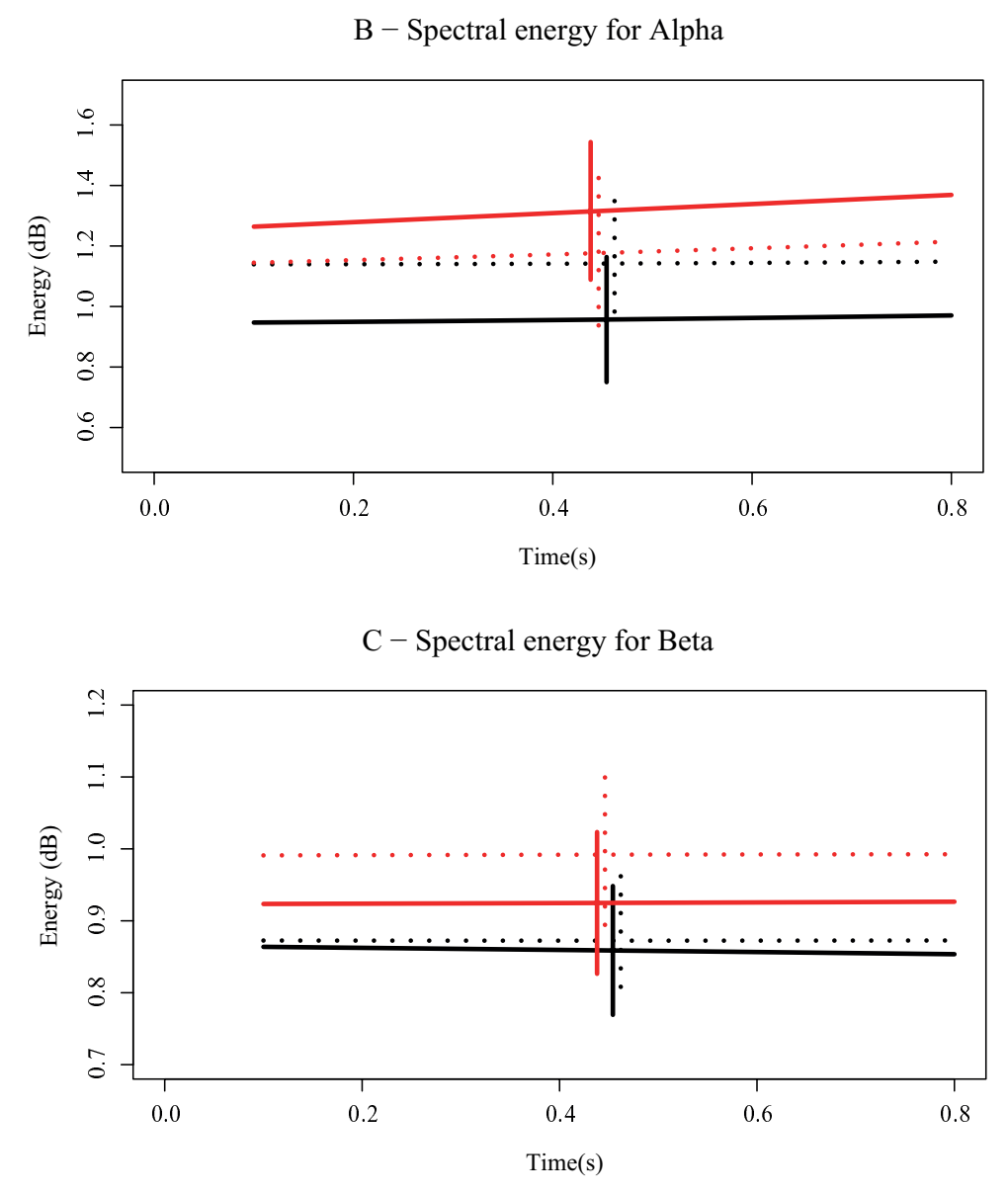

C - Spectral energy for Beta 
Figure 5
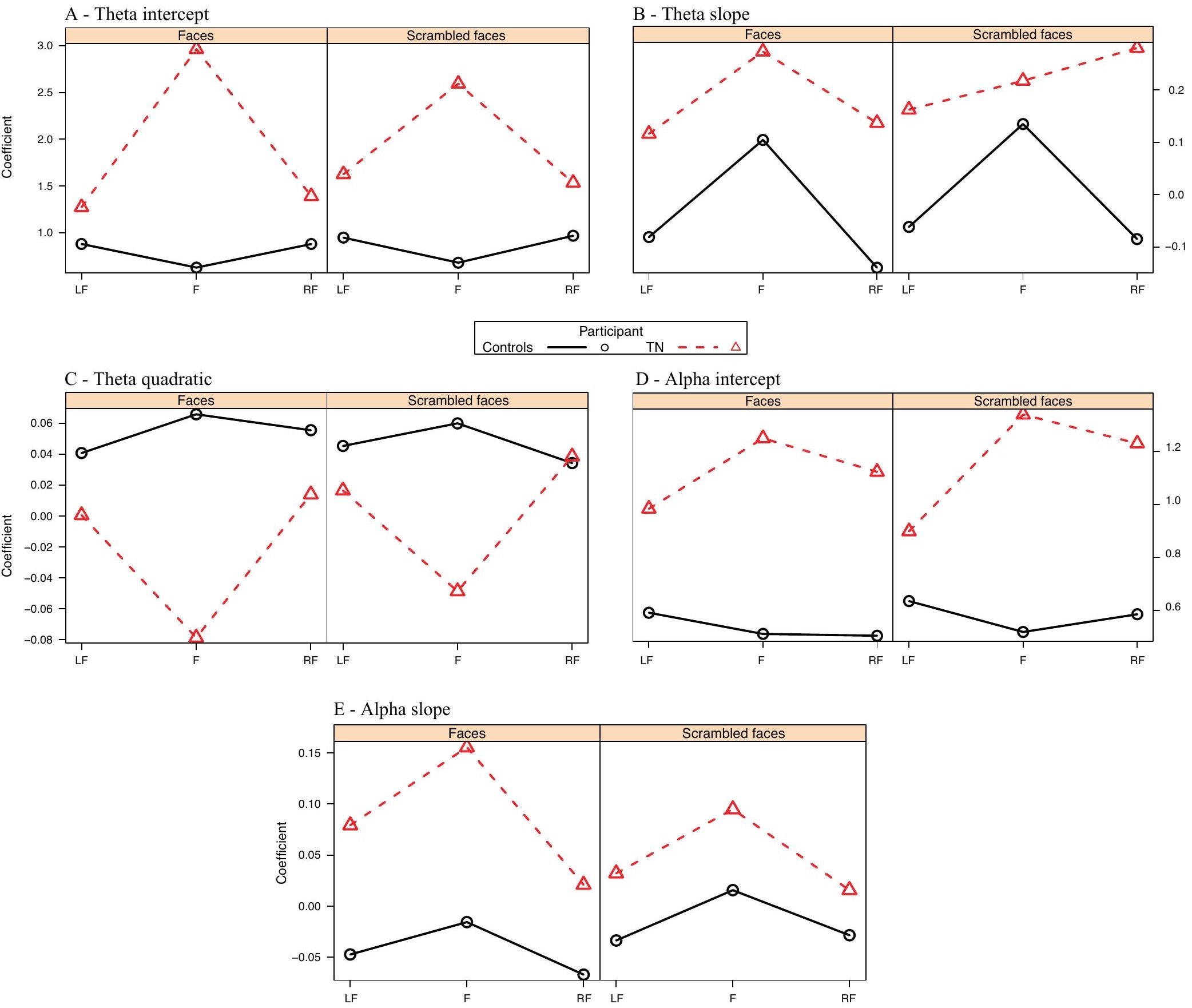
Table 1. Results of the t-tests

\begin{tabular}{lll} 
p-value & $\begin{array}{l}\text { p-value } \\
\text { Bonferroni } \\
\text { corrected }\end{array}$ \\
\hline
\end{tabular}

\section{Theta band}

Frontal

$$
\mathrm{t}(80)=6.329<10^{-5} * * *
$$

$<10^{-4} * * *$

Right frontal

$\mathrm{t}(80)=4.236$

$<10^{-4} * * *$

$<10^{-3} * * *$

Left frontal

$\mathrm{t}(80)=3.842$

$<10^{-4} * * *$

$<10^{-3} * *$

Alpha band

Frontal

$\mathrm{t}(80)=2.949$

$0.004 * *$

$0.036^{*}$

Right frontal

$\mathrm{t}(80)=1.626$

0.108

1

Left frontal

$\mathrm{t}(80)=2.152$

$0.034 *$

0.306

\section{Beta band}

\begin{tabular}{llll} 
Frontal & $\mathrm{t}(80)=-0.786$ & 0.435 & 1 \\
Right frontal & $\mathrm{t}(80)=-1.118$ & 0.267 & 1 \\
Left frontal & $\mathrm{t}(80)=-0.486$ & 0.628 & 1 \\
\hline
\end{tabular}

t-tests associated to the differences in the ERSP between the pre- $(-400-0 \mathrm{~ms})$ and post- $(100-800 \mathrm{~ms})$ stimulus period for each frequency band (theta, alpha, beta) and each region of interest (frontal, right frontal, left frontal) in TN. A positive t-test value indicates a post-stimulus ERSP increase; a negative t-test value indicates a post-stimulus ERSP decrease. The uncorrected and Bonferroni corrected pvalues associated to each test are also represented. 
Table 2. Results of the 9 ANOVAs

\begin{tabular}{|c|c|c|c|c|c|}
\hline & Theta intercept & Theta slope & Theta quadratic & Alpha intercept & Alpha slope \\
\hline \multicolumn{6}{|l|}{$\mathrm{C}$} \\
\hline Reg & $<10-5(<10-3)$ & & $0.045(0.045)$ & $0.0373(0.04964)$ & $0.01(0.043)$ \\
\hline Acc & $0.038(0.044)$ & $0.043(0.043)$ & & & $<10-3 \quad(0.0024)$ \\
\hline \multicolumn{6}{|l|}{$C^{*} \operatorname{Reg}$} \\
\hline $\mathrm{C} * \mathrm{Acc}$ & & & $<10-3(0.0087)$ & & \\
\hline \multicolumn{6}{|l|}{$\operatorname{Reg} *$ Acc } \\
\hline $\mathrm{C} * \operatorname{Reg} * \mathrm{Acc}$ & & & 0.0341 & & \\
\hline
\end{tabular}

Significant p-values associated with each parameter (intercept, slope, quadratic effect) in the three frequency bands (theta, alpha, beta) for the principal effects of each factor and their interactions: $\mathrm{C}=$ condition (faces/scrambled faces); Reg = region (left frontal/frontal/ right frontal); Acc = accuracy (correct/incorrect). The corrected p-values following the closure principle are displayed in brackets. Alpha quadratic, beta intercept, beta slope and beta quadratic colons are not shown as they display no significant results. 
Table 3. Results of the 9 multi-level models

\begin{tabular}{|c|c|c|c|c|c|c|c|}
\hline & Theta intercept & Theta slope & Theta quadratic & Alpha intercept & Alpha slope & Beta intercept & Beta slope \\
\hline \multicolumn{8}{|l|}{$\mathrm{C}$} \\
\hline $\mathrm{R}$ & $<10-15(<10-14)$ & $<10-6(<10-5)$ & $0.013(0.039)$ & $0.016(0.048)$ & $<10-5(<10-4)$ & & \\
\hline $\mathrm{P}$ & $0.005(0.015)$ & $0.017(0.051)$ & & $0.032(0.096)$ & & & \\
\hline $\mathrm{C} * \mathrm{R}$ & $0.034(0.102)$ & & & & & $0.004(0.012)$ & \\
\hline $\mathrm{C} * \mathrm{P}$ & & & & & $0.017(0.051)$ & & \\
\hline $\mathrm{R} * \mathrm{P}$ & $<10-16(<10-15)$ & $0.007(0.021)$ & $<10-5(<10-4)$ & $<10-6(<10-5)$ & & & $0.043(0.129)$ \\
\hline $\mathrm{C} * \mathrm{R} * \mathrm{P}$ & & & & & & $0.005(0.015)$ & \\
\hline
\end{tabular}

Significant p-values associated with each parameter (intercept, slope, quadratic effect) in the three frequency bands (theta, alpha, beta) for the principal effects of each factor and their interactions: $\mathrm{C}=$ condition (faces/scrambled faces); $\mathrm{R}$ = region (left frontal/frontal/ right frontal); $\mathrm{P}=$ participant (TN/controls). The corrected p-values are displayed in brackets. Alpha quadratic and beta quadratic colons are not shown as they display no significant results. 